Proc. of the 11 Int. School on Theoretical Physics Symmetry and Structural Properties of Condensed Matter, Rzeszów 2014

\title{
Symmetry-Induced Hybridization in Hexagonal and Zinc-Blende CrTe Crystals
}

\author{
M. KuZMA* \\ University of Rzeszów, Faculty of Mathematics and Natural Sciences, S. Pigonia 1, 35-959 Rzeszów, Poland

\begin{abstract}
Transition metal chalcogenides of a zinc-blende structure are theoretically predicted to be half metallic and, therefore, they are interesting for spintronic applications. However, the ground state of these compounds is hexagonal, of NiAs type, whereas the cubic phase is a metastable one. Here, we investigate the $\mathrm{Cr}-\mathrm{Te}$ bondings of both phases of $\mathrm{Cr}-\mathrm{Te}$ from point of view of symmetry. The hybridization in octahedral and tetrahedral lattice is studied for both structures. The fiber bundle presentation of the hybridization problem is also addressed.
\end{abstract}

DOI: $10.12693 /$ APhysPolA.128.185

PACS: 61.50.Ah, 75.50.Cc, 75.50.Pp

\section{Introduction}

Transition metal chalcogenides show metallic or semiconducting properties, depending on the anions and the ratio between the metal and chalcogen atoms. Moreover, these compounds exhibit various types of magnetic properties for different structures and compositions: ferromagnetic, antiferromagnetic or non-collinear spin arrangement. The $\mathrm{Cr}-\mathrm{Te}$ system is particularly interesting, while - among other $\mathrm{Cr}$ chalcogenides - only $\mathrm{CrTe}$ shows ferromagnetic properties at room temperature (e.g. CrSe, $\mathrm{CrS}$ are antiferromagnets) [1]. It forms several crystal phases $\mathrm{Cr}_{1-x} \mathrm{Te}$ of a hexagonal NiAs type crystal structure. Much attention has been devoted to half-metallicity of $\mathrm{CrTe}$. However, this property is discovered in $\mathrm{Cr}-\mathrm{Te}$ zinc-blende (ZB) only, which is not a stable phase of $\mathrm{CrTe}$. In paper [2], the stability of transition-metal pnictides ( $\mathrm{MnAs}, \mathrm{CrAs}, \mathrm{CrSb}$ ) has been studied by first principles methods. From the calculations, it follows that the energy of ZB phases of pnictides exceeds the energy of the ground state NiAs phases by about $1 \mathrm{eV} /$ f.u. In the case of chalcogenides, this difference of energy is about two or three times smaller [13 ], which makes this compounds preferable for the experimental fabrication of proper (room Curie temperature, adequate thickness of epitaxial layers, stability against distortions, etc.) half-metallic ferromagnets for spintronic applications. Some attempts to stabilize the ZB phase of chalcogenides have been proposed using the following methods: (1) choosing a substrate with a coherent lattice constant for epitaxial growth of the layers [4]; (2) pressure inducing of crystal and magnetic structure of material [5,6]; doping with magnetic or nonmagnetic ions [7]. However, the stabilization of the ZB phase of chalcogenides is still an open task. We investigate the properties of the chemical bonds in both structures of the $\mathrm{NiAs}$ type phases and the zinc-blende one. The results

\footnotetext{
*e-mail: kuzma@ur.edu.pl
}

make it possible to design a new method for growth of the ferromagnetic ZB phase of CrTe. Therefore, the aim of this paper is a group-theoretical analysis of the hybrids forming two types of clusters: tetrahedral and octahedral ones, which form the simplest clusters of the cubic and hexagonal phases of $\mathrm{CrTe}$, respectively. Moreover, based on the Racah-Wigner approach and the formalism of the action of a symmetry group on a set $[8,9]$, we treat the hybrids as a fiber bundle.

\section{Crystal structure of cubic and hexagonal $\mathrm{CrTe}$}

$\mathrm{Cr}_{1-x}$ Te systems crystallize in monoclinic hexagonal NiAs structure [10,11], with $\mathrm{Cr}$ atoms at the sites $(0,0,0)$ and $(0,0,1 / 2)$, and Te atoms at the sites $(1 / 3,2 / 3,1 / 4)$ and $(2 / 3,1 / 3,3 / 4)$ (Fig. 1a). The elementary cell is presented in Fig. 1a. Cr atoms, as well as Te atoms, form hexagonal layers separately, and they are arranged perpendicularly to the $z$ axes, at the distance $c / 2(c=$ $6.222 \AA$ ). Each $\mathrm{Cr}$ atom is surrounded by six $\mathrm{Cr}$ atoms in the $x y$ plane, at the distance $a=3.997 \AA$. Along the $z$ axes, the distance $\mathrm{Cr}-\mathrm{Cr}$ is shorter, equal to $c / 2$. a)

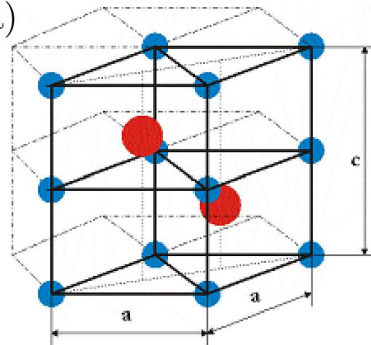

b)

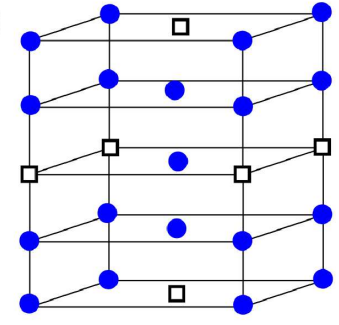

Fig. 1. Crystal structure of NiAs and the elementary cell of CrTe (a), crystal structure of the Cr sublattice of $\mathrm{Cr}_{2} \mathrm{Te}_{3}$ (b).

The deviation of stoichiometry in the CrTe system, shown by the index $x$ in $\mathrm{Cr}_{1-x} \mathrm{Te}$, results in various phases with the same symmetry, but a great number of 
the $\mathrm{Cr}$ vacancies. Non-stoichiometric phases may crystallize in the trigonal space group $P 3 m$. As an example, the arrangement of $\mathrm{Cr}$ atoms and its vacancies in the $\mathrm{Cr}_{2} \mathrm{Te}_{3}(x=0.33)$ crystal lattice is shown in Fig. $1 \mathrm{~b}$. The experimentally determined - through the X-ray diffraction (XRD) measurements - crystal data of more popular phases of CrTe have been collected in Table I. The main physical properties of particular phases are indicated in this table as well.

TABLE I

$\mathrm{XRD}$ and magnetic data of the $\mathrm{Cr}_{1-x}$ Te phases. Crystal parameters are taken from crystal data cards: a - ASTM 00-050-1153, b - ASTM 01-086-2500, c - ASTM 01-086-2506, d - ASTM 65-2312 B, e - ASTM 71-2245, f - ASTM 00-050-1153, g - ASTM 65-6816. Magnetic data are taken from papers of Dijkstra et al. [10, 11].

\begin{tabular}{|c|c|c|c|c|c|c|c|c|}
\hline \multirow[b]{2}{*}{ composition } & \multicolumn{4}{|c|}{$\mathrm{CrTe}$} & $\mathrm{Cr}_{3} \mathrm{Te}_{4}$ & $\mathrm{Cr}_{2} \mathrm{Te}_{3}$ & $\mathrm{Cr}_{5} \mathrm{Te}_{8}$ & $\mathrm{Cr}_{5} \mathrm{Te}_{6}$ \\
\hline & 0 & $0-0.13$ & & & $0.13-0.31$ & \multicolumn{3}{|c|}{0.33} \\
\hline crystal structure & cub. & hex. 1 & hex. 2 & hex. 3 & monoc. & hex. & $\begin{array}{c}\text { hex. } \\
\text { monoc. [1] } \\
\text { trig. [1] }\end{array}$ & hex. \\
\hline$a[\AA]$ & 6.29 & 3.90 & 6.82 & 3.99 & 6.84 & 6.814 & 7.815 & 13.935 \\
\hline$b[\AA]$ & & 3.90 & 6.82 & 3.99 & 3.89 & 12.073 & 7.815 & 3.954 \\
\hline$c[\AA]$ & & 6.00 & 12.07 & 6.22 & 12.53 & 1.77 & 11.989 & 6.857 \\
\hline$c / a$ & & 1.53 & 1.76 & 1.55 & 1.83 & & 1.53 & 2.03 \\
\hline$\alpha$ [deg] & & 90 & 90 & 90 & 91.13 & & 90 & \\
\hline$\beta$ [deg] & & 90 & 90 & 90 & & & 90 & \\
\hline$\gamma$ [deg] & & 120 & 120 & 120 & & & 120 & \\
\hline & [3] & [a] & [b] & {$[c]$} & [d] & [e] & {$[\mathrm{f}]$} & [g] \\
\hline exchange splitting $\mathrm{Cr} 3 \mathrm{~d}$ between $\uparrow \downarrow[\mathrm{eV}]$ & & 2.87 & & & 2.82 & 2.74 & & \\
\hline magnetic moment $\left[\mu_{\mathrm{B}}\right]$ & & & & 3.51 & 3.32 & 3.03 & & \\
\hline$T_{\mathrm{c}}[\mathrm{K}]$ & $327[12]$ & 340 & & & $315-340$ & 117 & & \\
\hline$N\left(E_{F}\right)[1 / \mathrm{eV}$ unit cell $]$ & & & & & $4.96 \uparrow, 1.08 \downarrow$ & & & \\
\hline
\end{tabular}

\section{Hybrids in cubic lattice of CrTe}

The zinc blende structure of a cubic lattice of $\mathrm{CrTe}$ consists of tetrahedral clusters in which each $\mathrm{Cr}$ atom is surrounded by four Te atoms, and vice versa (Fig. 2). These two kinds of atoms are connected together by hybrid bonds of a $\sigma$ and $\pi$ type. In Fig. 2, the $\sigma$ hybrids are depicted as atom bonds $\sigma_{1}, \sigma_{2}, \sigma_{3}, \sigma_{4}$.

The symmetry of the crystal structure presented in Fig. 2 is $T_{d}$ (Table II). The same symmetry is revealed in the system of hybrids $\sigma_{i}, i=1, \ldots, 4$. These hybrids form a $\Gamma_{\sigma}$ representation of the group, which is equivalent to the positional representation $P$ of Te atoms marked by the numbers $i=1, \ldots 4$. Aiming to determine the symmetry of the $\pi$ hybrids, the local reference system in each position of the Te atom is established, so that the $z$ axis in each node $n$ is directed towards the $\mathrm{Cr}$ atom. The characters of the $\Gamma_{\sigma}, \Gamma_{\pi}$ representations have been collected in Table II.

The decomposition of the reducible representations $\Gamma_{\sigma}, \Gamma_{\pi}$ into an irreducible one is as follows:

$$
\begin{aligned}
& \Gamma_{\sigma}=A_{1}+T_{2}, \\
& \Gamma_{\pi}=E+T_{1}+T_{2} .
\end{aligned}
$$

In the $T_{d}$ group, the atomic orbitals $s, p, d$ transform according to these representations, as in Table III:

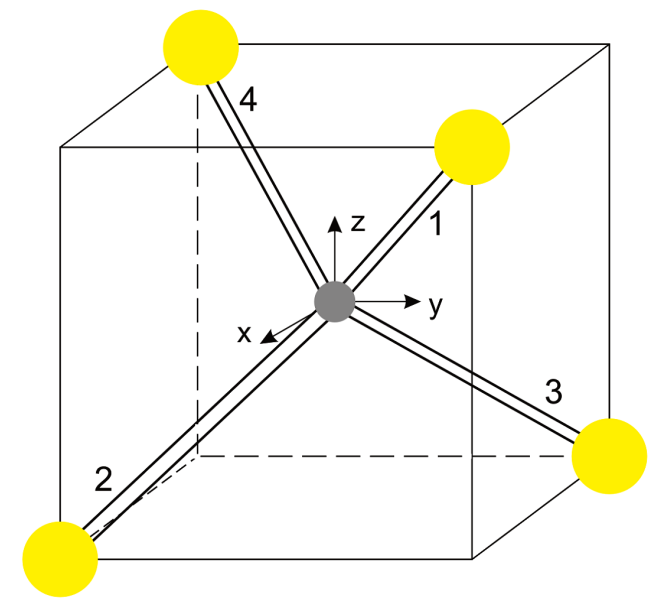

Fig. 2. Tetrahedral structure of the hybrids in zincblende CrTe.

$$
\begin{array}{ll}
s & A_{1} \\
p_{x}, p_{y}, p_{z} & T_{2} \\
d_{x y}, d_{x z}, d_{y z} & T_{2} .
\end{array}
$$

Taking into account the electron configuration of $\mathrm{Cr}$ and Te atoms (Table IV), the hybrids of the $\sigma$ type in cubic CrTe are $s p^{3}$, or $s d^{3}$. The lowest $d$ orbitals 
of $\mathrm{Cr}$ atoms are $3 d$ and the lowest $p$ orbitals of $\mathrm{Te}$ are $5 p$. Therefore, the hybridization is $p d$ and it shows a $T_{2}$ symmetry.

TABLE II

Irreducible representations of $T_{d}$ group and the $\Gamma_{\sigma}$ and $\Gamma_{\pi}$ hybrid representations.

\begin{tabular}{c|c|c|c|c|c}
\hline \hline$T_{d}$ & $E$ & $8 C_{3}$ & $3 C_{2}$ & $6 S_{4}$ & $6 \sigma$ \\
\hline$A_{1}$ & 1 & 1 & 1 & 1 & 1 \\
$A_{2}$ & 1 & 1 & 1 & -1 & -1 \\
$E$ & 2 & -1 & 2 & 0 & 0 \\
$T_{1}$ & 3 & 0 & -1 & 1 & 1 \\
$T_{2}$ & 3 & 0 & -1 & -1 & 1 \\
$\Gamma_{\sigma}$ & 4 & 1 & 0 & 0 & 2 \\
$\Gamma_{\pi}$ & 8 & 0 & 0 & 0 & 2
\end{tabular}

TABLE III

Transformation of the $s, p$ and $d$ atomic orbitals, according to irreducible representations of the $D_{3 h}, D_{3 d}, T_{d}$ and $O_{h}$ groups.

\begin{tabular}{c|c|c|c|c|c}
\hline \hline Orbitals & $D_{3 h}$ & $D_{3 d}$ & Orbitals & $T_{d}$ & $O_{h}$ \\
\hline$s$ & $A_{1}^{\prime}$ & $A_{1 \mathrm{~g}}$ & $s$ & $A_{1}$ & $A_{1 \mathrm{~g}}$ \\
$p_{x}$ & $E^{\prime}$ & $E_{\mathrm{u}}$ & $p_{x}$ & $T_{2}$ & $T_{1 \mathrm{u}}$ \\
$p_{y}$ & & & $p_{y}$ & & \\
$p_{z}$ & $A_{2}^{\prime \prime}$ & $A_{2 \mathrm{u}}$ & $p_{z}$ & & \\
$d_{x z}$ & $E^{\prime \prime}$ & $E_{\mathrm{g}}$ & $d_{x z}$ & $T_{2}$ & $T_{2 \mathrm{~g}}$ \\
$d_{y z}$ & & & $d_{y z}$ & & \\
$d_{x y}$ & $E^{\prime}$ & $E_{\mathrm{g}}$ & $d_{x y}$ & & \\
$d_{x^{2}-y^{2}}$ & & & $d_{x^{2}+y^{2}}$ & & \\
$d_{x^{2}+y^{2}}$ & $A_{1}^{\prime}$ & $A_{1 \mathrm{~g}}$ & $d_{x^{2}-y^{2}}$ & $E$ & $E_{\mathrm{g}}$ \\
$d_{z^{2}}$ & $A_{1}^{\prime}$ & $A_{1 \mathrm{~g}}$ & $d_{z^{2}}$ & &
\end{tabular}

TABLE IV

Electron configuration of $\mathrm{Cr}, \mathrm{Cd}$, and Te atoms.

\begin{tabular}{c|c|c|c}
\hline \hline Atom & No. of electrons & Configuration & $\begin{array}{c}\text { Excited } \\
\text { configuration }\end{array}$ \\
\hline $\mathrm{Cr}$ & 24 & $1 s^{2} \ldots 3 d^{5} 4 s$ & $4 p$ \\
$\mathrm{Cr}^{2+}$ & 22 & $1 s^{2} \ldots 3 d^{4}$ & \\
$\mathrm{Te}$ & 52 & $1 s^{2} \ldots 5 p^{4}$ & $5 d$ \\
$\mathrm{Cd}$ & 48 & $1 s^{2} \ldots 5 d^{10} 5 s^{2}$ & $6 p$
\end{tabular}

\section{Hybrids in hexagonal lattice of CrTe}

The hexagonal structure of CrTe is presented in Fig. 3.

Each Te atom is surrounded by six $\mathrm{Cr}$ atoms forming a cluster with a $D_{3 h}$ symmetry (Fig. 4a).

Aiming to form hybrids of a $\sigma$ and $\pi$ type between Te and $\mathrm{Cr}$ atoms, in each node of the $\mathrm{Cr}$ atom in Fig. 4a, a local frame system is established, with the $z$ axis directed towards the central atom. The $\sigma$ and $\pi$ hybrids form reducible representations of the $D_{3 h}$ group. They have been collected in Table Va.

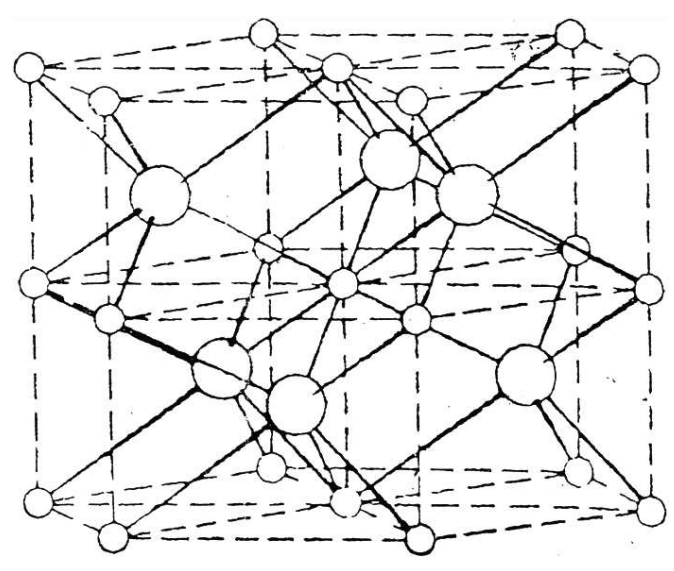

Fig. 3. Hexagonal structure of $\mathrm{CrTe}$ (small balls represent $\mathrm{Cr}$ atoms, $r_{\mathrm{Cr}}=1.223 \AA$, large balls are Te atoms, $r_{\mathrm{Te}}=2.037 \AA$ ).

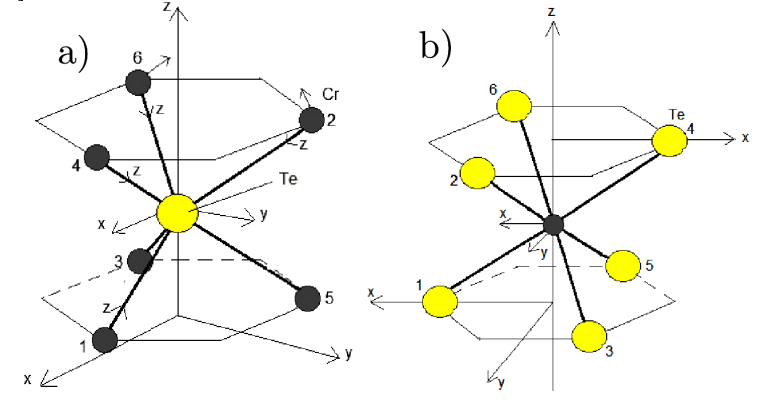

Fig. 4. Symmetry $D_{3 h}$ and $D_{3 d}$ of the nearest neighbourhood of $\mathrm{Te}$ (a) and $\mathrm{Cr}$ (b) atoms, respectively, in hexagonal CrTe.

TABLE V

Characters of the $D_{3 h}$ (a) and $D_{3 d}$ (b) groups and the characters of the $\Gamma_{\sigma}$ and $\Gamma_{\pi_{x}}, \Gamma_{\pi_{y}}$ representations.

\begin{tabular}{|c|c|c|c|c|c|c|c|}
\hline \multicolumn{8}{|c|}{ (a) } \\
\hline$C_{3 v}$ & $D_{3 h}$ & $E$ & $2 C_{3}$ & $3 C_{2}$ & $\sigma_{h}$ & $2 S_{3}$ & $3 \sigma_{d}$ \\
\hline$A_{1}$ & $A_{1}^{\prime}$ & 1 & 1 & 1 & 1 & 1 & 1 \\
\hline$A_{2}$ & $A_{2}^{\prime}$ & 1 & 1 & -1 & 1 & 1 & -1 \\
\hline$E$ & $E^{\prime}$ & 2 & -1 & 0 & 2 & -1 & 0 \\
\hline & $A_{1}^{\prime \prime}$ & 1 & 1 & 1 & -1 & -1 & -1 \\
\hline & $A_{2}^{\prime \prime}$ & 1 & 1 & -1 & -1 & -1 & 1 \\
\hline & $E^{\prime \prime}$ & 2 & -1 & 0 & 2 & 1 & 0 \\
\hline & $P$ & 6 & 0 & 0 & 0 & 0 & 2 \\
\hline & $\Gamma_{\pi x}$ & 6 & 0 & 0 & 0 & 0 & -2 \\
\hline & $\Gamma_{\pi y}$ & 6 & 0 & 0 & 0 & 0 & 2 \\
\hline \multicolumn{8}{|c|}{ (b) } \\
\hline & $D_{3 d}$ & $E$ & $2 C_{3}$ & $3 C_{2}$ & $i$ & $2 S_{6}$ & $3 \sigma_{d}$ \\
\hline & $A_{1 \mathrm{~g}}$ & 1 & 1 & 1 & 1 & 1 & 1 \\
\hline & $A_{2 \mathrm{~g}}$ & 1 & 1 & -1 & 1 & 1 & -1 \\
\hline & $E_{\mathrm{g}}$ & 2 & -1 & 0 & 2 & -1 & 0 \\
\hline & $A_{1 \mathrm{u}}$ & 1 & 1 & 1 & -1 & -1 & -1 \\
\hline & $A_{2 \mathrm{u}}$ & 1 & 1 & -1 & -1 & -1 & 1 \\
\hline & $E_{\mathrm{u}}$ & 2 & -1 & 0 & -2 & 1 & 0 \\
\hline & $\Gamma_{\sigma}$ & 6 & 0 & 0 & 0 & 0 & 2 \\
\hline & $\Gamma_{\pi x}$ & 6 & 0 & 0 & 0 & 0 & -2 \\
\hline & $\Gamma_{\pi y}$ & 6 & 0 & 0 & 0 & 0 & 2 \\
\hline
\end{tabular}


Decomposition of the hybrid representations into irreducible representations of the $D_{3 h}$ group is

$$
\begin{aligned}
& \Gamma_{\sigma}=P=A_{1}^{\prime}+A_{2}^{\prime \prime}+E^{\prime}+E^{\prime \prime}, \\
& \Gamma_{\pi x}=A_{2}^{\prime}+A_{1}^{\prime \prime}+E^{\prime}+E^{\prime \prime}, \\
& \Gamma_{\sigma y}=A_{1}^{\prime}+A_{2}^{\prime \prime}+E^{\prime}+E^{\prime \prime} .
\end{aligned}
$$

The nearest surrounding of the Cr atoms (Fig. $4 \mathrm{~b}$ ) is different with respect to the neighborhood of Te. The symmetry of the $\mathrm{CrTe}_{6}$ cluster is $D_{3 d}$. In this group, the $\Gamma_{\sigma}$ and $\Gamma_{\pi}$ hybrid representations have been collected in Table $\mathrm{Vb}$. Their decomposition is

$$
\begin{aligned}
& \Gamma_{\sigma}=P=A_{1 \mathrm{~g}}+A_{2 \mathrm{u}}+E_{\mathrm{g}}+E_{\mathrm{u}}, \\
& \Gamma_{\pi x}=A_{2 \mathrm{~g}}+A_{1 \mathrm{~g}}+E_{\mathrm{g}}+E_{\mathrm{u}}, \\
& \Gamma_{\sigma y}=A_{1 \mathrm{~g}}+A_{2 \mathrm{u}}+E_{\mathrm{g}}+E_{\mathrm{u}} .
\end{aligned}
$$

Choosing a new reference system $X Y Z$ in Fig. 4b, with $Z$ axis passing through $\mathrm{Cr}$ atoms, e.g. No. 2 and No. 5, the cluster $\mathrm{CrTe}_{6}$ forms octahedron and its symmetry is much reacher $\left(O_{h}\right.$ - see Fig. 5).

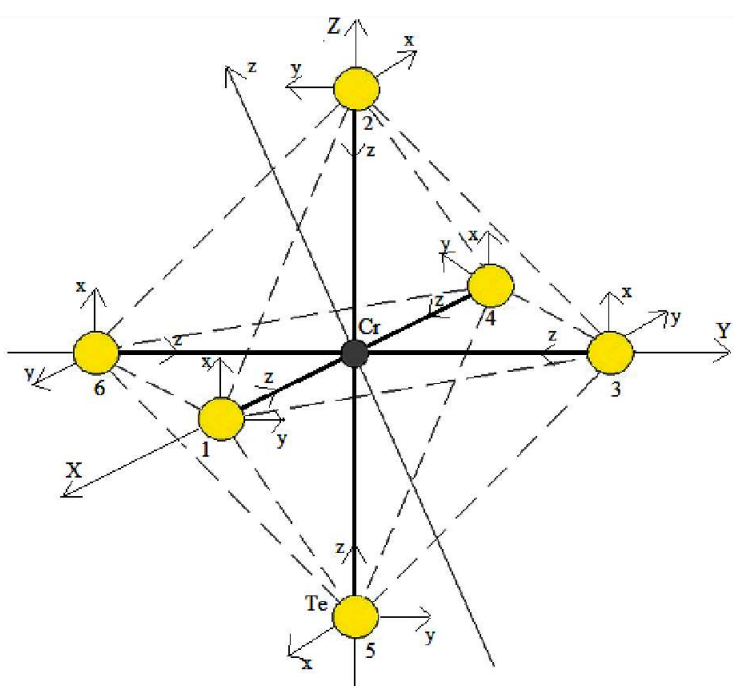

Fig. 5. Octahedral structure of the Te atoms surrounding $\mathrm{Cr}$ atoms in $\mathrm{CrTe}$.

In this case, for a proper description of the hybrids, it is convenient to provide - in each node of Te atoms - a local reference system, with the $Z$ axis directed towards the central atoms, as it was done in the previous examples. In such a symmetry group, it is not possible to divide the $\Gamma_{\pi}$ representation into two representations $\Gamma_{\pi x}$ and $\Gamma_{\pi y}$, while the $x$ and $y$ vectors do not form two separate orbits of the $O_{h}$ group. Hybrid representations are collected in Table VI.

Decomposition of the $\Gamma_{\sigma}$ and $\Gamma_{\pi}$ representations is as follows:

$$
\begin{aligned}
& \Gamma_{\sigma}=A_{1 \mathrm{~g}}+E_{\mathrm{g}}+E_{1 \mathrm{u}}, \\
& \Gamma_{\pi}=T_{1 \mathrm{~g}}+T_{2 \mathrm{~g}}+T_{1 \mathrm{u}}+T_{2 \mathrm{u}} .
\end{aligned}
$$

\section{TABLE VI}

Characters of the irreducible representations of the $O_{h}$ group group and the characters of the $\Gamma_{\sigma}$ and $\Gamma_{\pi}$ representations.

\begin{tabular}{c|c|c|c|c|c|c|c|c|c|c}
\hline \hline$D_{h}$ & $E$ & $8 C_{3}$ & $6 C_{2}$ & $6 C_{4}$ & $3 C_{4}^{2}$ & $i$ & $6 S_{4}$ & $8 S_{6}$ & $3 \sigma_{h}$ & $6 \sigma_{d}$ \\
\hline$A_{g}$ & 1 & 1 & 1 & 1 & 1 & 1 & 1 & 1 & 1 & 1 \\
$A_{2 \mathrm{~g}}$ & 1 & 1 & -1 & -1 & 1 & 1 & -1 & 1 & 1 & -1 \\
$E_{\mathrm{g}}$ & 2 & -1 & 0 & 0 & 2 & 2 & 0 & -1 & 2 & 0 \\
$T_{1 \mathrm{~g}}$ & 3 & 0 & -1 & 1 & -1 & 3 & 1 & 0 & -1 & -1 \\
$T_{2 \mathrm{~g}}$ & 3 & 0 & -1 & 1 & -1 & 3 & 1 & 0 & -1 & -1 \\
$A_{1 u}$ & 1 & 1 & 1 & 1 & 1 & -1 & -1 & -1 & -1 & -1 \\
$A_{2 u}$ & 1 & 1 & -1 & -1 & 1 & -1 & 1 & -1 & -1 & 1 \\
$E_{u}$ & 2 & -1 & 0 & 0 & 2 & -2 & 0 & 1 & -2 & 0 \\
$T_{1 u}$ & 3 & 0 & -1 & 1 & -1 & -3 & -1 & 0 & 1 & 1 \\
$T_{2 u}$ & 3 & 0 & 1 & -1 & -1 & -3 & 1 & 0 & 1 & -1 \\
\hline$\Gamma_{\sigma}$ & 6 & 0 & 0 & 2 & 2 & 0 & 0 & 0 & 4 & 2 \\
$\Gamma_{\pi}$ & 12 & 0 & 0 & 0 & -4 & 0 & 0 & 0 & 0 & 0
\end{tabular}

\section{Fiber structure of hybrids}

The positions of atoms in the $R$ cluster are nodes $1,2,3, \ldots, N$.

$$
R=\{1, \ldots, N\} .
$$

The nodes form a positional space $P[8]$ :

$$
P=L C\left\{e_{r} \mid r \in R\right\},
$$

so that $P$ is the space of all the linear combinations of the nodes over the $C$ field of complex numbers.

In each node, there is an atom having atomic orbitals $(A O)$. These form a single-centre $A O$ space. The configurational space $L$ of the system is a tensor product

$$
L=P \otimes A O .
$$

The configuration space $L$ can be illustrated by a fiber bundle. Let the nodes $r \in R$ be described by a function of the unitary space $W_{r}$. The isomorphism $\varphi_{r}: W \longrightarrow W_{r}$ maps a certain model space $W$ onto $W_{r}$. Let $E$ be a sum

$$
E=\cup_{r \in R} W_{r} .
$$

The structure $(E, R, W, p)$ is fiber bundle, with $R$ being the base space, and $W$ being the standard fiber. The fiber bundle scheme of $p$ hybrids in a molecule consisting of 9 atoms is presented in Fig. 6.

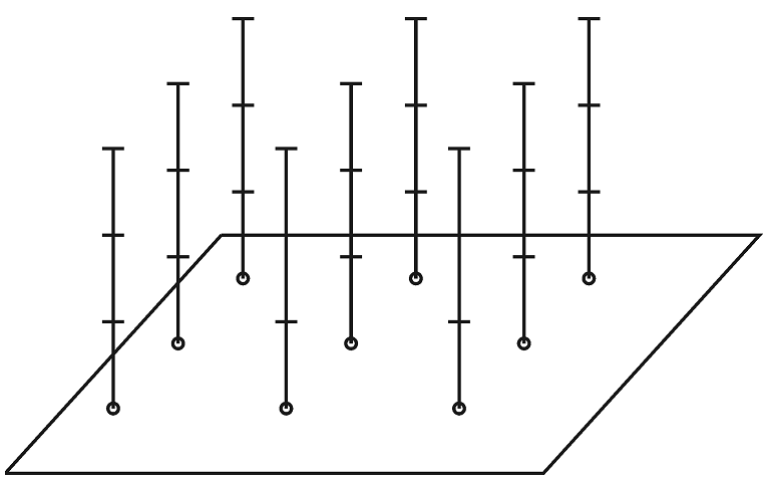

Fig. 6. Fiber bundle model of the hybrids in a molecule consisting of 9 atoms. 
The action of the symmetry group $G$ of a cluster in the configurational space $L$ forms the mechanical representation $M$, which is the product of

$$
M=R \otimes V,
$$

where $R$ is a transitive representation of the group $G$, determined by the stability group $H \subset G$ of node 1 ,

$$
R=R^{G: H} .
$$

$V$ is the vector representation in an atomic orbital $(A O)$ space. Both representations, i.e. $R$ and $V$, are reducible and may be decomposed into irreducible representations of the symmetry group $G$ :

$$
\begin{aligned}
& R=\Sigma_{\Lambda} m_{R, \Lambda} \Lambda, \\
& V=\Sigma_{\Delta} m_{V, \Delta} \Delta,
\end{aligned}
$$

where $m_{R, \Lambda}$ and $m_{V, \Delta}$ are multiplicity factors.

\section{Results and discussion}

According to Table III, in the $O_{h}$ and $T_{d}$ group, the $s, p, d$ orbitals of $\mathrm{Cr}$ atom are transformed as

$\begin{array}{lll} & O_{h} & T_{d} \\ s & A_{1 \mathrm{~g}} & A_{1} \\ d_{x 2-y 2}, d_{z^{2}} & E_{\mathrm{g}} & E \\ p_{x}, p_{y}, p_{z} & T_{1 \mathrm{u}} & T_{2} \\ d_{x y}, d_{x z}, d_{y z} & T_{2 \mathrm{~g}} & T_{2} .\end{array}$

TABLE VII

\begin{tabular}{|c|c|c|c|c|c|c|c|c|c|c|}
\hline \multirow{2}{*}{ sym. } & $\mathrm{Cr}$ & $\mathrm{Te}$ & \multirow[b]{2}{*}{$N$} & \multicolumn{6}{|c|}{ Te ligand number } & \multirow[b]{2}{*}{ hyb. } \\
\hline & \multicolumn{2}{|c|}{$\begin{array}{l}\text { atomic } \\
\text { orbital }\end{array}$} & & \multicolumn{2}{|c|}{\begin{tabular}{|l|l|}
1 & 2
\end{tabular}} & 3 & \multirow[t]{2}{*}{\begin{tabular}{l|l}
3 & 4
\end{tabular}} & \multirow[t]{2}{*}{5} & \multirow[t]{2}{*}{6} & \\
\hline \multicolumn{8}{|c|}{$O_{h}$} & & & \\
\hline \multirow[t]{2}{*}{$A_{1 \mathrm{~g}}$} & \multirow[t]{2}{*}{$s$} & & \multirow{2}{*}{$\frac{1}{\sqrt{2 \cdot 3}}$} & 1 & 1 & 1 & 1 & 1 & 1 & $s \sigma$ \\
\hline & & $p_{z}$ & & 1 & 1 & 1 & 1 & 1 & 1 & $p \sigma$ \\
\hline \multirow{2}{*}{$E_{\mathrm{g}}$} & $d_{z 2}$ & $s$ or $p_{z}$ & $\frac{1}{2 \sqrt{3}}$ & -1 & 2 & -1 & -1 & 2 & -1 & $s \sigma$ \\
\hline & $d_{x 2-y 2}$ & 28 & $\frac{1}{2}$ & 1 & & 1 & 1 & & -1 & $p \sigma$ \\
\hline \multirow{3}{*}{$T_{1 \mathrm{u}}$} & $p_{x}$ & & \multirow{3}{*}{$\frac{1}{\sqrt{2}}$} & \multirow[t]{3}{*}{1} & \multirow[b]{3}{*}{1} & \multirow{3}{*}{\multicolumn{2}{|c|}{-}} & & \multirow{3}{*}{-1} & \multirow[t]{3}{*}{$s \sigma$} \\
\hline & $p_{y}$ & $s$ or $p_{y}$ & & & & & & & & \\
\hline & $p_{z}$ & $p_{z}$ & & & & & & -1 & & \\
\hline \multirow{3}{*}{$T_{2 \mathrm{~g}}$} & $d_{x z}$ & & \multirow{3}{*}{$\frac{1}{2}$} & $p_{y}$ & $p_{x}$ & & $p_{x}$ & $p_{y}$ & & \\
\hline & $d_{y z}$ & $p_{y}, p_{x}$ & & & $p_{y}$ & $p_{x}$ & & $p_{x}$ & $p_{y}$ & $p d \pi$ \\
\hline & $d_{x y}$ & & & $p_{x}$ & & $p_{y}$ & $p_{y}$ & & $p_{x}$ & \\
\hline \multicolumn{11}{|c|}{$T_{d}$} \\
\hline \multirow[t]{2}{*}{$A_{1}$} & \multirow[t]{2}{*}{$s$} & $s$ & \multirow{2}{*}{$\frac{1}{2}$} & 1 & 1 & 1 & 1 & & & \\
\hline & & $p_{z}$ & & 1 & 1 & 1 & 1 & & & \\
\hline \multirow{2}{*}{$E$} & $d_{z 2}$ & $p_{x}$ & \multirow{2}{*}{$\frac{1}{2}$} & 1 & -1 & -1 & 1 & & & \\
\hline & $d_{x^{2}-y^{2}}$ & $p_{y}$ & & 1 & -1 & -1 & 1 & & & \\
\hline \multirow{3}{*}{$T_{2}$} & & $p_{x}$ & & 1 & 1 & -1 & -1 & & & \\
\hline & $s$ or $p_{2}$ & $p_{y}$ & $\frac{1}{2}$ & 1 & -1 & 1 & -1 & & & $\operatorname{sp} \sigma$ \\
\hline & & $p_{z}$ & & 1 & -1 & -1 & 1 & & & \\
\hline & $d_{y z}$ & $p_{x}+p_{y}$ & $\frac{1}{4}$ & 1 & -1 & 1 & -1 & & & \\
\hline & & & $\frac{\sqrt{3}}{4}$ & -1 & $1-1$ & 1 & & & & \\
\hline$T_{2}$ & $d_{x z}$ & $p_{x}+p_{y}$ & $\frac{1}{4}$ & 1 & 1 & -1 & -1 & & & $p d \pi$ \\
\hline & & & $\frac{\sqrt{3}}{4}$ & 1 & 1 & -1 & -1 & & & \\
\hline & $d_{x y}$ & $p_{x}$ & $-\frac{1}{2}$ & 1 & 1 & 1 & $\mid 1$ & & & \\
\hline
\end{tabular}

Symmetry orbitals for the octahedral and tetrahedral clusters of CrTe (sym. - symmetry, hyb. - type of hybrydization, $N$ - normalization factor).
The set of atom orbitals of ligands, i.e. six Te atoms in an octahedron (having $6 \times 4 s p$ orbitals $=24$ ), as well as the set of orbitals of four Te atoms in a tetrahedron $(4 \times 4 s p$ orbitals $=16)$, should have the same symmetry properties.

The symmetry orbitals (symmetry basis of the representations) of the type $A, E, T_{1}, T_{2}$ for both clusters have been calculated by the projection method, and are collected in Table VII. They correspond to the hybrids studied. The bases for the $T_{1 \mathrm{u}}$ representation forming hybrids $\pi$ have been omitted, while the $p_{x}, p_{y}$ orbitals of ligands interact with the $p$ orbitals of the central ion very weakly.

\section{Conclusions}

We have provided a comparison of the symmetry properties of $\mathrm{Cr}$-Te hybrid bonds in two different structures of CrTe: a hexagonal one, of a NiAs type, and a cubic one, of a zinc-blende type. It has been noticed that in a hexagonal structure, the bonds form an octahedron $\mathrm{CrTe}_{6}$. In a cubic structure, the analogue cluster is a tetrahedron $\mathrm{CrTe}_{4}$. The coincidence as regards the symmetry of the hybrids for both structures has been shown. Moreover, a similarity between the bases of the hybrids for both structures is an unexpected result. From these studies, we conclude that the symmetry of the zinc-blende phases of $\mathrm{CrTe}$ is not the main reason for the difficulty in growing zinc-blende $\mathrm{CrTe}$.

\section{References}

[1] I. Galanakis, P. Mavropoulos, Phys. Rev. B 67, 104417 (2003).

[2] B. Sanyal, L. Bergqvist, O. Eriksson, Phys. Rev. B 68, 054417 (2003).

[3] W.-H. Xie, Y.-Q. Xu, B.-G. Liu, Phys. Rev. Lett. 91, 037204 (2003).

[4] Y.-J. Zhao, A. Zunger, Phys. Rev. B 71, 132403 (2005).

[5] V. Kanchana, G. Vaitheeswaran, M. Rajagopalan, J. Magn. Magn. Mater. 250, 353 (2002).

[6] T. Eto, M. Ishizuka, S. Endo, T. Kanamata, T. Kikegawa, J. Alloys Comp. 315, 16 (2001).

[7] K. Nakamura, T. Ito, A.J. Freeman, Phys. Rev. B 72, 064449 (2005).

[8] T. Lulek, Acta Phys. Pol. A 57, 407 (1980).

[9] T. Lulek, M. Szopa, J. Phys. A Math. Gen. 23, 677 (1990).

[10] J. Dijkstra, H.H. Weitering, C.F. van Bruggen, C. Haas, R.A. de Groot, J. Phys. Condens. Matter 1, 9141 (1989).

[11] J. Dijkstra, C.F. van Bruggen, C. Haas, R.A. de Groot, J. Phys. Condens. Matter 1, 9163 (1989).

[12] M.G. Sreenivasan, K.L. Teo, M.B.A. Jalil, T. Liew, T.C. Chong, A.Y. Du, IEEE Trans. Magn. 42, 2691 (2006). 